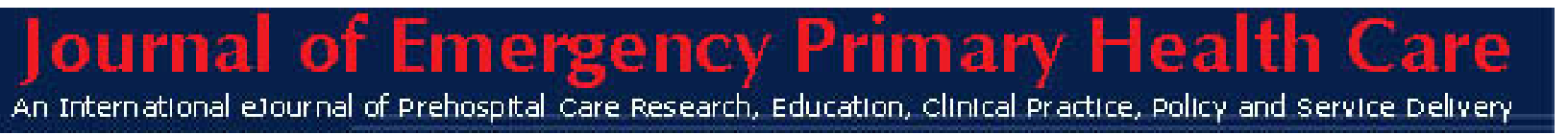

ISSN 1447-4999

\title{
ABSTRACTS
}

\section{Australian College of Ambulance Professionals (ACAP) 2005 National Conference}

'Something for Everyone'

Canberra, Australia $13^{\text {th }}$ to $16^{\text {th }}$ October 2005

The Management Committee gratefully acknowledges the support of the Australian College of Ambulance Professionals, and all authors who have submitted ACAP National Conference 2005 abstracts to the Journal of Emergency Primary Health Care for publication.

The Management Committee would also like to thank Toby Keene for his support and contribution in the collection, administration and preparation of the abstracts for submission the the journal. 


\section{Is Mechanism of Injury Only a Useful Predictor in Prehospital Trauma Triage} Mal Boyle, Erin Smith, lan Patrick, Tony Walker, \& Frank Archer

Abstract 990125-01

Background: The Review of Trauma and Emergency Services in Victoria (1999) left unresolved the predictive value of mechanism of injury in pre-hospital trauma triage guidelines. International literature is controversial, with previous studies focusing on major trauma defined at hospital and neglecting the pre-hospital perspective where these important triage decisions are made.

Aims: The research question was: "Is mechanism of injury a useful predictor in pre-hospital trauma triage?"

Methods: All Victorian ambulance trauma Patient Care Records (PCRs) for 2002 were reviewed. PCRs that had mechanism of injury only trauma were identified and compared with the State Trauma Registry to determine those who sustained retrospectively defined major trauma. Data was stored in a Microsoft Access database, cleaned and loaded into statistical software for analysis. Ethics committee approval was obtained.

Results: There were 53,360 trauma incidents, of these $7.7 \%$ were mechanism of injury only. $62 \%$ were males and $38 \%$ females, median age was 28 years. The majority of patients with mechanism of injury only were involved in an MCA $>60 \mathrm{~km} / \mathrm{hr}(\mathrm{n}=1,822)$, of these only $9(0.5 \%)$ sustained retrospectively defined major trauma. The other main mechanisms were motor cyclist/cyclist $(n=l, 459)$, of whom $0.2 \%$ sustained major trauma, patients in a vehicular rollover $(n=524)$ of whom none sustained major trauma, and a fall from greater than $5 \mathrm{~m}(\mathrm{n}=38)$ of whom $5.2 \%$ sustained major trauma.

Conclusions: The study suggests that individual mechanism of injury criteria have no statistical, clinical, or operational significance in prehospital trauma triage when used as mechanism of injury only criteria, ie. in those trauma patients who are not physiologically distressed, nor have a significant pattern of injury as defined. That there is sufficient evidence to support the changes to pre-hospital trauma triage as included in the current Ambulance Victoria Clinical Practice Guidelines, ie. to exclude mechanism of injury only from the criteria for prehospital major trauma. These results add to the knowledge base of trauma presentation in the pre-hospital setting, especially in Australia, and are the baseline for further studies. 


\title{
A twelve month profile of trauma in Victoria for 2002 - an ambulance perspective
} Mal Boyle, Erin Smith, lan Patrick, Tony Walker, \& Frank Archer

\begin{abstract}
990125-02
Background: The Review of Trauma and Emergency Services in Victoria (1999) left unresolved the predictive value of mechanism of injury in pre-hospital trauma assessment. International literature is controversial, with previous studies focusing on Major Trauma defined at hospital and neglecting the pre-hospital perspective where these important triage decisions are made.
\end{abstract}

Aims: The research question was: "What is the pre-hospital trauma profile in Victoria, from an ambulance perspective?"

Methods: All Victorian ambulance trauma Patient Care Records (PCRs) for 2002 were reviewed. PCRs were excluded if they did not meet the study criteria. Data was stored in a Microsoft Access database, cleaned and loaded into statistical software for analysis. Ethics committee approval was obtained.

Results: There were 53360 trauma incidents. Of the patients transported, 67\% were males and 33\% females, median age was 30 years. $1.4 \%$ were in physiological distress and $21 \%$ had a significant pattern of injury. 9\% had a significant mechanism of injury without physiological distress or significant pattern of injury. Standing falls accounted for 39\%, minor trauma $18 \%$, and DOAs $0.6 \%$. $7 \%$ were not transported. Some patients falling from less than five metres or who fell from skateboards etc, were noted to have suffered significant injury.

Conclusions: This profile is unique in the literature as it represents a total population for a twelve month period of ambulance response to trauma. The results add to the knowledge base of trauma presentation in the pre-hospital setting and are the baseline for analysis of the mechanism of injury only sub-group. 


\section{Acute medication related events attended by ambulance paramedics in Metropolitan Melbourne \\ Paul Dietze, Jennie Hutton, Penny Heale, Stefan Cvetkovski, Andrew Dent \& Stephen Burgess}

Abstract 990125-03

Objective: To describe the characteristics of non-fatal medication related ambulance attendances in Melbourne from December 1998 to September 2002.

Methods: A retrospective analysis of 16,705 patient care records (PCRs) completed by ambulance paramedics in greater metropolitan Melbourne in which medications were identified as having a causal role in the attendance.

Results: The mean age of cases was 35 years and $61 \%$ were female. A single medication only was implicated in 11,765 cases $(70 \%$ of the total). Of these, $85 \%$ involved one of six types of medication: benzodiazepines (52\%), paracetamol (15\%), selective serotonin re-uptake inhibitors (SSRIs) (6.5\%), combination paracetamol and opioids (4\%), phenothiazines (3.4\%) and tricyclic anti-depressants (TCAs) (3.7\%). This presentation will detail the descriptive epidemiology of cases and summarize the statistical analysis of the influence of the various combinations of the pharmacology ingested. Patterns of patient clinical presentation will be discussed.

Conclusion: The implications of the data for paramedic clinical practice will be discussed. Although management may require only simple supportive treatment and patients may not be transported to hospital, the ongoing medical and psychiatric treatment of the significant number of patients not transported to hospital in the study period needs to be considered. Special attention will be given to the factors that influence a reduced presenting Glasgow Coma Score (GCS) in non-fatal medication overdose patients. 


\title{
Heatwave mitigation in Queensland \\ Peter Chatman
}

\begin{abstract}
990125-04
Heatwave related illness and death is a worldwide issue. In Australia, heatwaves have caused more deaths than any other natural disaster but people are unaware of the dangers ${ }^{l}$. Most at risk are the very young, the chronically ill and elderly. ${ }^{2}$
\end{abstract}

Apathy and Ignorance is a real killer. Surprisingly, despite thousands of heatwave deaths worldwide, little has been done to prepare in Australia. Regardless of the reason, apathy makes these events all the more dangerous. ${ }^{3}$

A few examples include the:

- $\quad$ "Black Friday" bushfires of Victoria caused 71 deaths and is still remembered, but the associated heatwave that claimed 438 lives almost went unnoticed ${ }^{4}$

- 2000 Southeast Queensland Heatwave directly caused 22 deaths and 350 hospitalisations ${ }^{5}$

- 2004 Southeast Queensland Heatwave directly caused 12 deaths and 221 hospitalisations 5

It is phenomenal that these heatwaves hardly raised an eyebrow within the communities or media. By comparison, 18 people died in the Thredbo landslide, ${ }^{6}$ and just 4 people died in the Canberra bushfires ${ }^{7}$ but both created a furore and are easily recalled.

Internationally, the death tolls from heatwaves are horrendous. The French heatwave in August 2003 killed 14,800 people. ${ }^{8}$ Even more shocking is that the European heatwave of the same period killed 35,118 people. ${ }^{8}$ This also had a cost impact of US $\$ 13$ billion, ${ }^{9}$ can we afford not to prepare?

Is Australia Prepared? Worldwide, some heatwaves have been catastrophes through poor policy and ill preparedness. ${ }^{10}$ This is a strong warning for those accountable to take note and mitigate what will occur in the future. There is a need for decisive action by governments for a resolve to 'unnecessary' heatwave deaths. ${ }^{3}$

Does your State or Territory have a mitigation plan for Heatwave? It's not a case of if it happens, just a matter of when! Most Heatwave deaths are 'preventable' and as such Queensland has undertaken a whole of government approach to heatwave preparedness.

It is imperative that mitigation is based on weather warnings relating to 'human health risk'. Australia has never had such a system, but the Commonwealth Bureau of Meteorology; Queensland division recently developed an interim measure. ${ }^{11}$ Outwardly, we should adopt best practice of Kalkstein's (Delaware USA) Heat Health Watch Warning System, which predicts hot weather health risk 5 days out and accounts for cloud cover, wind, acclimatisation, social structure, and urban landscape of each city it is applied to. ${ }^{10}$ Thus it accounts for varying health reactions of people from one city to the next. No other system does this. ${ }^{12}$ Philadelphia USA has this model in place and studies using three years of data has confirmed that 117 lives were saved by their heat program. ${ }^{13}$

Within QAS, staff health is a critical concern in a heatwave. In 2004, three QAS paramedics were hospitalised and many others showed signs of heat stress. If paramedic health is not addressed, there is a 
risk of having no officers available to provide services. ${ }^{3}$ Following past experiences, the QAS has implemented systems to mitigate this.

Southeast Queensland now has a Heatwave Strategy underpinned by the Department of Emergency Services/QAS Heatwave Response Plan. This was implemented on 17th December 2004.

This presentation will provide a gripping insight into heatwaves, global events, risk, and the various hot weather-warning systems. It will provide an overview of the Queensland experience of the 2004 heatwave and the processes undertaken in developing the whole of government strategy and systems implemented within the QAS Heatwave Emergency Response Plan.

\section{References:}

1. Australian Red Cross, 2004. Latest News. Heatwaves, Australia's deadliest 'hidden' natural hazard. http://.redcross.org.au/?fuseaction=NEWSROOM.archive\&sub=334

2. Centres for Disease Control and Prevention. CDC, 14th May 2004.Emergency Preparedness and Response. Extreme Heat. Viewed $4^{\text {th }}$ September 2004, online. http: //www. bt. cdc. gov/disaster s/extremeheat/about. asp

3. Chatman PA. November $17^{\text {th }}$, 2004. Heatwave Preparedness In Queensland, Disaster by Stealth and Death By Apathy.

4. Emergency Management Australia 2001. Hazards, Disasters and Survival, A Booklet for Students and the Community. Heatwave Hazards and Disasters. Viewed 4th September 2004, online, http ://www. csu. edu. au/faculty/health/aemf/HD S/chapter_4.htm

5. Queensland Heat Wave Response Plan. Draft Version 1, pp 4, December 2004

6. Aussurvivalist, $30^{\text {th }}$ June 2004. Natural Hazards and Disasters in Australia, Geological and extraterrestrial hazards, overview. http://www.aussurvivalist.com/naturalhazards.htm

7. Australian Capital Territory Legislative Assembly, Tabling Statement 2003. Report of the Inquiry into the Operational Response to the January 2003 Bushflres in the ACT. Delivered by Jon Stanhope MLA Chief Minister, 19 $9^{\text {th }}$ August 2003. Viewed $4^{\text {th }}$ September 2004, Online. http://www.cmd.act.gov.au/mcleod_inquiry/Documents/Tabling.doc

8. Larsen J, Earth Policy Institute. October 2003. Record heat wave in Europe takes 35,000 lives. Far Greater Losses May Lie Ahead. Viewed 4th September 2004, online. http://www.earthpolicy.org/Updates/Update29.htm

9. Long S. 2003. ABC Radio Transcript, Natural Disasters Costing Insurance companies. http://www.abc.net.au/am/content/2003/sl018Q22.htm

10. Ford P. November $7^{\text {th }}$, 2003. French Holiday Sacrificed. The Christian Science Monitor. Viewed 4th September 2004, online, http://www.csmonitor.com/2003/1107/p06s02-woeu.html 
11. BerginM. September 14*2004. Commonwealth Bureau Of Meteorology, Queensland Division. Heatwave Warning Strategy, Thresholds.

12. Sheridan SC, Kalkstein LS. 2004, Progress in heat watch-warning system technology. Accepted in the Bulletin of the American Meteorological Society to appear December 2004.

13. Johnson DL. National Oceanic and Atmospheric Administration, NOAA. National Weather Service, NOAA Heat/health watch warning system improving forecasts and warnings for excessive heat. Excessive heat program piloted in Philadelphia is becoming worldwide model.

http: '/www. noaanews. noaa.sov/stories2005/s2366. htm 


\title{
Developments in pre-hospital delivery of thrombolysis by ambulance paramedics
} Mark E. Cooke

\begin{abstract}
990125-05
Intravenous therapy with thrombolytic agents is an established treatment for AMI with about 50,000 uses each year in the UK.

The introduction of newer bolus thrombolytic has greatly simplified the practicalities of prehospital delivery, enabling patients to achieve the benefits associated with early thrombolysis. Bolus interventions form part of the NICE guidance for the use of thrombolytic drugs and were recommended for use in the prehospital phase (technology appraisal 52, October 2002).

The East Midlands Ambulance Service (EMAS) were the first to introduce the prehospital administration of thrombolysis (PHT) by paramedics, using the double bolus agent reteplase; at present (February 2005), 27 of the 31 English NHS ambulance services provide PHT in accordance with the Joint Royal Ambulance Liaison Committee (JRCALC) clinical guidelines, and 2 further services are evaluating alternative methods for prompt revascularisation.
\end{abstract}

By end December 2004, 1659 patients had received PHT (either tenecteplase or reteplase), of which 1310 have been within the last 12 months.

The mean 'Call to Needle' time for this group of patients was less that 40 minutes, which demonstrates that paramedics are adequately equipped and educated to acquire and interpret 12lead ECGs, accurately identify patients suitable for thrombolysis, and well placed to administer the therapy. This is usually conducted in conjunction with decision support by means of telemetry and mobile phones, but as confidence grows more and more ambulance services are moving towards autonomous practice. Recent developments within MINAP and CCAD together with the development of a specific ambulance outcome database, clinical outcome will soon be correlated with PHT.

There is no evidence to suggest that there have been any adverse incidents directly attributed to prehospital administration of thrombolysis, either in terms of inappropriate administration or major haemorrhage. This safety record reflects the strict guidelines set out by JRCALC and the cautious approach taken by paramedics.

Although at present PHT accounts for less than 5\% of all patients thrombolysed each year, much progress has been made, with the number of patients receiving PHT rising substantially each month.

The delivery of PHT can reduce ‘call to needle’ times substantially and should be supported. 


\title{
Randomised comparison of the SLIPA ${ }^{\mathrm{TM}}$, (Streamlined Liner of the Pharynx Airway), and the SS-LM (Soft Seal Laryngeal Mask) by health professional trainees \\ Cindy Hein, John Plummer, \& Harry Owen
}

\begin{abstract}
990125-06
Background and Goal of the Study: The need to establish a patent airway is a vitally important skill for paramedics where experience and patient exposure may limit success. The Laryngeal Mask Airway has been well established as a device that can be used even by those who are novices [1-3]. The SLIPA ${ }^{\mathrm{TM}}$ is a new supraglottic airway device that has previously been shown to be easy to insert by medical officers and anaesthetists of varying experience [4]. Additionally, the SLIPA ${ }^{\mathrm{TM}}$ requires less steps for placement as it does not require cuff inflation and has a "bite-block" within its shaft. These features suggest that the SLIPA $^{\mathrm{TM}}$ may be a useful airway device for inexperienced users.

This randomised crossover trial compared the SLIPA ${ }^{\mathrm{TM}}$ (Hudson) and the SS-LM (Portex) in terms of success rates, time taken for insertion and user preference, when inserted by novice users.

Methods: Ethics committee approvals were obtained and the Therapeutic Goods Administration was informed of a multi-centre trial. Thirty-six medical students who had not used or been trained to use either device were trained in the airway laboratory and assessed by OSCE (Objective Structured Clinical Exam). The students then inserted each device, in randomised sequence, in two separate patients with only 2 insertion attempts per patient allowed. Patients were excluded if they were under the age of 18 years, had not given informed consent, had illnesses associated with delayed gastric emptying, morbid obesity, risk of pulmonary aspiration or were pregnant. Outcome measures were; success or failure; success at first attempt and time to ventilation. The primary outcome was, which device did the students prefer to use.

Results: The SLIPA ${ }^{\mathrm{TM}}$ was successfully inserted (1 or 2 attempts) in 94\% of patients (34/36) and the SSLM in $89 \%$ (32/36). $1^{\text {st }}$ attempt success rates were 83\% (30/36) and 67\% (24/36) in the SLIPA ${ }^{\mathrm{TM}}$ and SSLM respectively. Median time to ventilation (removal of facemask to chest inflation) was 41.6 seconds (22.1 - 236) with the SLIPA ${ }^{\mathrm{TM}}$ and 55 seconds with the SS-LM (31.1- 233.3) (p .014). Sixty-seven percent of the students preferred to use the SLIPA ${ }^{\mathrm{TM}}$.

Conclusions: In this study, students demonstrated high success rates with both devices even though they were novice users. The shorter times to ventilation in the SLIPA ${ }^{\mathrm{TM}}$ group may prove to be of clinical significance, particularly in the emergency setting.

Whilst the Laryngeal Mask has gained wide recognition for use by both novice users and as a rescue airway in failed intubation, the data presented here suggests that the SLIPA ${ }^{\mathrm{TM}}$ may also prove useful in these areas. The challenge now, would be to access its potential use within the pre-hospital environment.
\end{abstract}

\section{References:}

1. Benumof, J.L., Laryngeal mask airway and the ASA difficult algorithm. Anesthesiology, 1996. 84(3): p. 686.

2. Choyce, A., et al., A comparison of the intubating and standard laryngeal mask airways for airway management by inexperienced personnel. Anaesthesia, 2001. 56(4): p. 357360.

3. Coulson, A., et al., A comparison of the ProSeal and Classic Laryngeal Mask Airways for airway management by inexperienced personnel after manikin-only training. Anaesth Intensive Care, 2003. 31: p. 286-289. 
4. Hein, C.L., H. Owen, and J. Plummer, The SLIPA ${ }^{\mathrm{TM}}$ - A disposable supraglottic device that is easy to use, in ASA Annual meeting, Las Vegas, October 2004, http://www.asahq.org/ 


\section{What is the role of the clinical instructor and clinical placements for Student Ambulance Paramedic training?}

Tina Ivanov

Abstract 990125-07

Pre-hospital research is a relatively new field, as in the past, most ambulance practice has been based on evidence extrapolated from general medical and hospital research. However, ambulance practice is proving to be a field of medicine requiring specific research and information for what is a dynamic, unpredictable and unique area of practice. Nevertheless, as the collection of prehospital research grows, the focus remains mainly on ambulance clinical practice, with the area of education in ambulance an extremely limited field. ${ }^{1-7}$ By exploring all areas of pre-hospital medicine, including the education of the participants, information can be gained on not only the most appropriate clinical practice, but also the most appropriate way to teach that practice.

As part of the requirements for completion of a Masters in Professional Education and Training (Deakin University), I am currently completing a research project that explores ambulance education. This project will be completed by September 2005, therefore a full summary of results would be available for presentation at the October ACAP conference.

The project aims to explore the role of the Clinical Instructor and clinical placements during the 'on-road' phase for Student Ambulance Paramedic (SAP) training in Rural Ambulance Victoria (RAV). Specifically, the project aims to investigate the knowledge, skills and attributes required by workplace Clinical Instructors, according to stakeholders, and examine the significant experiences, extent of knowledge and skills consolidated, and perceived benefits of participating in clinical placements. The stakeholders are those people with direct links to the on-road training program, and include the Clinical Instructors, Student Ambulance Paramedics, and the clinical 'supervisors' which includes Clinical Support Officers, Qualified Ambulance Paramedic Team Managers, Acting General Manager Paramedic Education and Training, and Manager Clinical Education and Development. The clinical placements explored in this project are limited to the 'on-road' phase of student training that includes direct supervision by a clinical instructor, while performing the duties of a paramedic.

The project consists of a mixed study of both qualitative and quantitative methodologies that allows for exploration of people's perceptions and experiences. Three groups of people will be invited to participate, namely the current Clinical Instructors within RAV, the Student Ambulance Paramedics, and the Clinical 'Supervisors'. Each individual of each group will be invited to complete a questionnaire. The results will be collated and analysed to provide details of the participants' ideas and experiences during the 'on-road' phase of clinical placements, and comment on the value of the current clinical placement system and the role of the Clinical Instructor.

Bibliography

1. Arneman, J. (1998) Current Practice and Developing Trends in the ALS Management of Cardiac Arrest. Australasian Journal of Emergency Care. Vol. 5 No. 4: ppl5-18. 
2. Ferrazzi, S., Waltner-Toews, D., Abernathy, T., and McEwan, S. (2001) The Effects of Prehospital Advanced Life Support Drug Treatment on Patient Improvement in Hospital Utilisation. Prehospital Emergency Care. Vol. 5 No. 3 pp252-260.

3. Grossman, D.C., Allegra, K., Macdonald, S.C., Klein, P., Copass, M.K., and Maier, R.V. (1997) Urban-Rural Differences in Prehospital Care of Major Trauma. Journal of TraumaInjury Infection and Critical Care. Vol. 42 No. 4 pp723-729.

4. Kilner, T. (2004a) Desirable Attributes of the Ambulance Technician, Paramedic, and Clinical Supervisor: Findings From a Delphi Study. Emergency Medicine Journal. Vol. 21 No. 3: pp374-378.

5. Kilner, T. (2004b) Educating the Ambulance Technician, Paramedic, and Clinical Supervisor: Using Factor Analysis to Inform the Curriculum. Emergency Medicine Journal. Vol. 21 No. 3: pp379-385.

6. Liberman, M., Mulder, D., and Sampalis, J. (2000) Advanced or Basic Life Support for Trauma: Meta-Analysis and Critical Review of the Literature. Journal of Trauma-Injury Infection and Critical Care. Vol. 49 No. 4 pp584-599.

7. Stout, J., Pepe, P.E., and Mosesso V.N. Jr. (2000) All-Advanced Life Support vs TieredResponse Ambulance Systems. Prehospital Emergency Care. Vol. 4 No. 1 ppl-6. 


\title{
Colour, markings and warning lights for emergency vehicles: A safer workplace or a flawed corporate vision John Killeen
}

\begin{abstract}
990125-09
Crews responding in emergency vehicles under urgent driving conditions rely on the warning systems built into their vehicles to assist in providing a safe path through traffic and around the incident scene after their arrival.

Many emergency service organisations have based their choice of design and colour (for warning lights and vehicle livery) on tradition, personal preference or as an opportunity to advertise their corporate image. Emergency vehicles throughout the world often unknowingly compromise safety by displaying inferior warning lights alongside unsuitable colours and patterns. These mixed visual messages may confuse other motorists, thus slowing their reaction times. As a consequence, the number of road accidents involving emergency vehicles continues to increase every year.
\end{abstract}

In future years, emergency service organisations will find themselves under increasing scrutiny from both the public and the judiciary following accidents involving agency vehicles. Most of the new research into emergency vehicle accidents has been driven by shifting OH\&S legislation and the threat of litigation. These changes to the level of accountability and responsibility within emergency agencies will directly affect their future policy decisions.

The problems of audible siren warnings being at odds with modern vehicle design will make evident the need to understand the physiology of vision, perception and human cognitive process, including how these factors can influence driver reaction times. Modifications to the colour and the livery of an emergency vehicle can significantly increase the situational presence of the responder to other drivers. The many aged, vision impaired or colour blind drivers on our roads benefit considerably from new enhanced passive and active warning systems fitted to emergency vehicles. Analysis of the optimum vehicle colour and pattern will consider the overseas experience and make comparisons with Australian vehicles while maintaining an emphasis on the local conditions. The capacity of some popular patterns to camouflage a vehicle will be assessed.

Retro-reflective fluorescent vehicle markings have provided the solution to the problems of effective passive warning. The benefits of different colours and types of warning lights (including the latest LED technology) will be described.

A blueprint for vehicle colour, markings and warning lights will outline a specification for the optimum vehicle layout. An understanding of human visual factors combined with the latest scientific research allows new strategies for passive and active warning systems to be established, leading to safer emergency vehicle operations for the future. 


\section{Pre-hospital intubating laryngeal mask airway trial Michael McCall}

Abstract 990125-10

Establishment of a secure airway is fundamental to the resuscitation of the critically ill and trauma patient. This is usually achieved in the pre-hospital setting by advanced airway-trained Paramedics using direct laryngoscopy and tracheal intubation. Paramedics without such training attempt to maintain oxygenation in critically ill patients using a bag-valve-mask assembly with or without an Oral (Guedel) airway until more skilled personnel and equipment are available.

Success rates for tracheal intubation by trained Paramedics are generally high ( $>90 \%$ in most reported series). However, these high success rates are mostly achieved by Paramedics in metropolitan areas with high clinical caseload and in patients in whom vital signs are absent and who seldom recover. The success rate in patients with vital signs (medical emergencies and trauma) is lower, reported at less than $75 \%$ in a recent study - one to examine this group. ${ }^{1}$

In trauma there is a high index of suspicion that the cervical spine may be damaged and so the neck is immobilised prior to any airway procedures. During laryngoscopy and intubation the head and neck are maintained in the neutral position by manual inline stabilisation (MILS) as recommended in established trauma programmes, such as the "Early Management of Severe Trauma" (EMST) program. Neck immobilisation makes facemask ventilation, laryngoscopy and intubation more difficult. ${ }^{2}$ The Intubating Laryngeal Mask Airway (ILMA - Fastrach) was designed to allow both ventilation and blind intubation after placement. It is best inserted with the head in neutral position (with one pillow) ${ }^{3}$ and only requires limited mouth opening. The insertion technique is easy to learn. A high success rate (91\%) in a mannequin has been demonstrated after 60 second training programme. In a study on cadavers ${ }^{5}$ inexperienced participants achieved adequate ventilation in $92 \%$ of the time on first insertion, higher than with a conventional laryngeal mask airway (LMA). The Intubating Laryngeal Mask Airway (ILMA) is easier to insert than the LMA in patients with MILS $S^{6}$. However, like the LMA, the presence of a stiff neck collar ${ }^{7}$ and cricoid pressure ${ }^{8}$ reduces the success of placement.

Most studies have demonstrated that adequate ventilation is more likely to be achieved with the ILMA or LMA than with conventional facemask ventilation (with or without an oral airway), ${ }^{4,59}$ Ambulance Officers and Paramedics will volunteer to be involved in ILMA training and the trial. The objective of this study is to evaluate the utilisation of the ILMA in the pre-hospital setting by Officers of the Tasmanian Ambulance Service in critically ill and trauma patients.

The participants in this trial will be the Ambulance Officers and Paramedics who volunteer to complete ILMA training and use the ILMA in the pre-hospital field. Ambulance Officers and Paramedics from around the state, with or without advanced airway training, may volunteer to be involved in the Prehospital Intubating Laryngeal Mask Airway Trial (PILMAT). After appropriate training, the participants will be able to use the ILMA on adult patients, greater than 50 kilograms in weight, who meet any one of the following inclusion criteria:

- Where airway management is required in an unconscious patient, or;

- Where assisted ventilation is required.

There will be no patient inclusion or exclusion based on their presenting clinical problem. 
The study is a prospective cross-sectional interventional study. The ILMA will be used as a first line airway in all patients identified by officers (non-intubators) as requiring an airway due to inadequate patency and/or respiration. Endotracheal intubation may be attempted via the ILMA by paramedics with advanced airway training (intubators) when the following criteria are satisfied:

- Intubation indicated as per protocols;

- Sedative drugs are given, if required, as per protocols;

- Ventilation via the ILMA is adequate.

Hypotheses for the PILMAT trial are:

1. In critically ill patients, the pre-hospital use of the intubating laryngeal mask airway will lead to improved ventilation by Ambulance Paramedics and improved patient outcomes.

2. Ambulance Paramedics prefer to use the intubating laryngeal mask airway over the bag-valvemask system to ventilate a patient.

3. The pre-hospital use of the intubating laryngeal mask airway, to intubate patients decreases their time to hospital arrival.

4. Ambulance Paramedics can be easily trained in the use of the intubating laryngeal mask airway with minimal skills maintenance requirements.

\section{References:}

1. Roeca B, Crosby E, Maloney J, Bryson G. An assessment of paramedic

2. performance during invasive airway management. Prehosp Emerg Care. 2000; 4(2):164-7.

3. Penant JH, Pace NA, Gajraji NM. Role of the laryngeal mask airway in the immobile cervical spine. J Clin Anesth. 1993; 5:226-30.

4. Kapila A, Addy EV, Verghese C, Brain AI. The intubating laryngeal mask airway: an initial assessment of performance. Br J Anaesth. 1997 Dec;79(6):710-3.

5. Levitan RM, Ochroch EA, Stuart S, Hollander JE. Use of the intubating laryngeal mask airway by medical and nonmedical personnel. Am J Emerg Med. 2000;18(l):12-6

6. Choyce A, Avidan MS, Patel C, Harvey A, Timberlake C, McNeilis N, Glucksrnan E. Comparison of laryngeal mask and intubating laryngeal mask insertion by the naïve intubator. $\mathrm{Br} J$ Anaesth. 2000;84(1): 103-5

7. Asai T, Wagle AU, Stacey M. Placement of the intubating laryngeal mask is easier than the laryngeal mask during manual in-line neck stabilization. Br J Anaesth. 1999;82(5):712-4

8. Wakeling $H$, Nightingale J. The intubating laryngeal mask airway does not facilitate tracheal intubation in the presence of a neck collar in simulated trauma. Br J Anaesth. 2000;84(2):254-6

9. Choyce A, Avidan MS, Shariff A, Del Aguila M, Radcliffe JJ, ChanT. A comparison of the intubating and standard laryngeal mask airways for airway management by inexperienced personnel. Anaesthesia 2001; 56:350-69.

\section{Additonal Reading}

Harry RM, Nolan JP. The use of cricoid pressure with the intubating laryngeal mask. Anaesthesia. 1999; 54(7):656-9. 


\section{Patient refusal of ambulance transport by case type: Queensland Ambulance Service 2004 Jamie Murdoch}

Abstract 990125-11

The literature in emergency medicine publications documents significant numbers of patients who refuse transport to hospital or primary care facilities after receiving ambulance treatment. Specifically, the literature provides evidence to suggest that particular types of cases are more likely to refuse transport than others.

The issue of patient transport refusal has fuelled debate about the risk of repeat episodes, lack of follow up, poor disease management and the costs associated with increased case acuity and the subsequent workload on Emergency Medical Services.

The Queensland Ambulance Service (QAS) observes a clear policy regulating the individual's right to refuse transport. For an individual to have the capacity to refuse transportation, they must do so voluntarily; be informed of the risks associated with refusal of transport; and have the mental capacity to do so. When paramedics are satisfied that the patient has the appropriate capacity to refuse transport, this individual right is observed.

Aim: The aim of this paper is to investigate the differences between cases where patients are transported to hospital, and those that refuse transport after being treated. Differences between the types of cases attended by QAS will be investigated, and any significant differences in patient recidivism reported.

Method: This study was a retrospective analysis of QAS patient data from 2004; where the patient refused transport after receiving treatment. The data used in the study were derived from the AIMS (QAS Ambulance Integrated Management System) database; compiled using original Ambulance Report Forms (ARF) to classify and code all QAS attended cases.

A literature review was also conducted to assess trends of transport-refusal in other Emergency Medical Services.

Discussion: The initial hypothesis was that a significant proportion of patients who refuse transport initially suffer from non-acute conditions that facilitate efficient responses from onscene paramedic treatment. Additionally, it was expected that individuals who fear legal or social consequences might also be likely to refuse transport and the resulting identification in the health system.

The results will be discussed in full at the presentation, together with a discussion on possible explanations for these phenomena and implications for Emergency Medical Services. 


\title{
Changing characteristics of drug overdose cardiac arrest in Queensland 2000-2003
}

Jamie Murdoch

\begin{abstract}
990125-12
Introduction: Harmful drug use has become a major concern within Australian society; one dimension of this concern is the increase in the number of deaths related to drug use. Illicit drug use is associated with a range of adverse effects on both physical and mental health. The incidence of misuse of prescription medications is also increasing, to represent a significant number of drug-induced deaths in Australia. In 2002 the Queensland Ambulance Service (QAS) responded to 1,641 cases of prescribed drug poisoning / overdose compared with 1,007 cases of recreational drug poisoning / overdose.
\end{abstract}

Aim: The aim of this poster is to investigate the most severe cases of drug overdose attended by the Queensland Ambulance Service between 2000 and 2003. Included in the study are 390 acute drug overdose cases attended by QAS paramedics between 2000 and 2003, where the overdose resulted in cardiac arrest.

Method: A retrospective analysis of QAS cardiac arrest data where drug poisoning / overdose was classified as the presumed aetiology of arrest. The data used in the study is derived from the Cardiac Outcomes Project database. This database uses AIMS (QAS Ambulance Integrated Management System) data and original Ambulance Report Forms (ARF) to classify cases and ensure coding is accurate.

Results: In $50 \%$ of cases the patient was found to be deceased and no resuscitation was commenced. Where resuscitation was attempted, $17.5 \%$ saw the return of spontaneous circulation on arrival at hospital.

The number of recreational drug overdoses almost halved between $2000(n=70)$ and $2001(n=36)$. Males accounted for $72.5 \%$ of acute recreational drug overdose in the four-year period 2000 2003. However, the proportion of females treated for acute prescribed drug overdose in the same period was greater, accounting for $52 \%$ of female cases overall, compared with $48 \%$ recreational drug overdoses.

There has been an increase in overall prescription drug overdoses from 2000, with a far greater percentage being pronounced "Dead on Arrival” (72\%) than recreational drug overdoses (55\%).

Individuals experiencing a cardiac arrest due to recreational drug overdose are slightly more likely to have been witnessed by a bystander or paramedic $(12.3 \%)$ than those suffering a prescription drug overdose (7.4\%). In addition, resuscitation attempts were more likely to be made with recreational drug overdose cases $(42.9 \%)$ than prescription drug overdose cases (27\%).

Discussion: These data show that very few drug-induced cardiac arrests are witnessed. This is a major contributing factor to the poor survival outcomes illustrated in the results. The data also suggests a slight increase in witnessed arrest and resuscitation attempts for recreational drug 
overdose. A greater proportion of recreational drug overdoses occur in public places, which may explain this phenomenon.

Prescription drug overdoses are more likely to occur in private residences; amongst older individuals; make up a greater proportion of female drug overdose cases; and are less likely to be witnessed. These data suggest that prescription drugs may be an avenue to suicide for some individuals, and is a growing proportion of all drug overdose incidents in Queensland. 


\title{
Jack of all trades, master of none LAC Brett Murrell
}

\begin{abstract}
990125-13
The presentation will explore the diverse role of an ADF Medical Assistant (Medic) whilst on deployment. From a strictly personal perspective the presentation will also take into account the psychological aspects that may have had an impact (both positive and negative) on the performance of these duties.

It is therefore the intention of the presenter to explore the psychological reactions experienced by personnel, including myself and how the medical related conditions and the environmental factors played a significant role in forming these emotions.

By explaining the various roles that I performed and by providing an honest account of my own psychological state. I hope to provide a candid description of my time spent in a combat zone.

The aim is to provide other health professionals with an insight into what they may encounter if faced with similar conditions. I believe that the broad training that I have received was adequate however it is the rapport that I was able to develop with the soldiers that enabled me to successfully fulfil my role.
\end{abstract}




\title{
Electrocardiography: A study of paramedics' learning methodologies
}

Madeleine O'Donnell

\begin{abstract}
990125-14
There is no record in EMS literature that describes how student paramedics learn to read the ECG. If we know about how students learn then instructors can modify their teaching practices in order to optimize student learning. When a person experiences a cardiac event, cardioversion and administration of life-saving drugs relies on accurate ECG interpretation. The aim of the study was to record cognitive functions that paramedics describe when they are learning to read the ECG. The study is positioned in the qualitative paradigm.
\end{abstract}

Research Question. What activities, strategies and cognitive elements do paramedics describe when they are learning to read ECG interpretations? 'Elements' are defined as those mental activities that are involved in student's thinking. Elements refer to a range of approaches, systems, concepts, or methodologies through which individuals say they learn.

Methods. Twelve participants were interviewed using semi-structured technique. This format allowed probing and prompting questions at the point of expression to extrapolate meaning. Data were coded into six themes with 51 sub-categories. Content analysis was concerned with description, induction, generation, and construction in terms of commonality in how ECG learning occurred.

Results. Interview transcripts articulated descriptions of students' cognitive learning events. Six themes emerged and were defined as:

BASIC LEARNING ACTIVITIES (8.3\%) - elementary skills derived from reading, writing, and calculating.

TRANSFORMA TION OF INFORM A TION PROCESSES (41.2\%) - comprehension originating from elementary skills. 3-dimensional visualizations and mental imagery was emphasized.

LEARNING INTERACTIONS (18\%) - events external to the classroom setting concerned with initiating, listening to, and participating in conversation.

CONTEXT OF LEARNING (14.5\%) - informal activities through clarification with selected peers.

SELF-REGULATORY LEARNING EVENTS (17.4\%) - students taking responsibility for their own learning.

AFFECTIVE LEARNING (6\%) - how students think and feel about their learning.

Conclusion. The subject matter of ECG interpretation is complex. Students undertaking such learning will, as part of their clinical practice, make life-and-death decisions based on actions from their learning. Therefore, it is imperative that students are given the support to learn, in a manner that is conducive to the way that the say they learn, hi response to this, instructors need to 
understand how students go about their learning and incorporate these processes into teaching their methods.

In this study each student provided their individual descriptions of how they went about their learning. Collectively, most of the student's descriptors were consistent with contemporary descriptions of learning. The data analysis has identified that students try to ensure their learning conforms to the given instruction. However they did not elaborate about their affective learning which suggests that they may or may not understand the importance of this approach. Each student identified their use of three-dimensional mental visualizations to be a contributing cognitive function, therefore, instructors need to consider incorporating this approach into instructional design as a way to optimize and strengthen students' learning about ECG interpretations. 


\section{Beyond Emergency Response.}

Peter O'Meara, Charles Sturt University, (on behalf of the research team)

Abstract 990125-15

This research project is being carried out during 2005 with funding from the Convention of Ambulance Authorities is using an industry partnership approach to review and analyse emerging roles of paramedics in regional and rural areas.

The study's objectives are to:

1. identify Australian and international trends in the evolving role of ambulance paramedics;

2. compare alternative models of ambulance practice that are emerging in rural and regional Australia;

3. determine the key characteristics, roles and expected outcomes for an ESP that are desirable, feasible and acceptable to key stakeholders;

4. identify the knowledge, skills and educational requirements necessary for ESP roles, and

5. develop an ESP model that assists ambulance services to set strategic directions in Australia.

The project is using a multiple case-study approach, where each of the extended paramedic roles in New South Wales, Victoria, South Australia and Tasmania are providing a distinct case for description and analysis of performance. In-depth nature data collection will allow inclusion of all relevant inter-professional interactions and important cultural factors in presenting these data.

As few locations within Australia have an ESP role the case studies selected for the study are distinctive examples of extended practice. The sampling framework incorporates one selected case per partner ambulance service. Data collection for each case was determined by the operational location of the paramedic; the timeframe of the research; and the interactions the paramedic has with other health professionals, health workers and community members within their ESP role.

Interim results and conclusions of the study will be presented. It is expected that they will set the strategic directions for an Expanded-Scope Paramedic (ESP) role for rural ambulance services in Australia. Expanding ambulance paramedics' scope of practice offers the potential to improve patient care and the general health of the community. The project also aims to increase the research capacity of Australian pre-hospital services. 


\title{
Graduate paramedic career choices: student intentions, influences and outcomes Peter O'Meara \& Veronica Madigan
}

\begin{abstract}
990125-16
The advent of university degrees in paramedic studies is changing the balance of power from ambulance authorities to students and graduates. Students and graduates can not only choose which ambulance authority they wish to seek employment with, they can also opt to enter related health professions through further study. Ambulance authorities now need to market themselves to prospective employees who have alternate options, whether that is with interstate competitors or other health professions.

This is a preliminary report of a longitudinal study being conducted over three years to establish the preferred destination of full-time paramedic students from Charles Sturt University. The principle purpose is to gain an understanding of the factors that drive graduate intentions and to suggest strategies that may encourage students to consider a career with the Ambulance Service of New South Wales.

Current students and graduates are being surveyed to ascertain the factors that shape their preferences for employment with Australian ambulance authorities or other employers.
\end{abstract}

The objectives of the research are to:

1. Determine the initial career intentions of full-time paramedic students and graduates and how they change over time during the paramedic course and after employment in industry.

2. Identify the key factors that influence student and graduate career choices.

3. Consider the implications of these finding and recommend policy changes that may have a positive impact on student career choices.

Questionnaires are being progressively distributed to those who have been enrolled as full-time students in the CSU paramedic program, whether they have completed the course full-time or have become distance education students following recruitment to ambulance authorities during the course. The questionnaires capture basic demographic details such as current enrolment and/or employment details, place of origin, gender and age. Current full-time students are being asked to nominate their current preferences for employment with potential employers. All respondents will be asked to identify the key factors which influenced their preferences in the domains of self, community-at-large, professional and organisational.

The findings from this study will generate recommendations related to the selection of students, the program of study at CSU, clinical placement and mentoring programs, and the recruitment programs and career pathways within the Ambulance Service of New South Wales. Other factors which may influence student preferences are their original place of origin, their own self-image, family influences, and content and quality of the education program. 